\title{
Use of the antenatal antiretroviral (ARV) tracking form in maternity case records to improve ARV management
}

\author{
T P Kerry, ${ }^{1}$ MB ChB, Dip Mid COG (SA), MFamMed; P G T Cudahy, ${ }^{2,3}$ MD \\ ${ }^{1}$ District Clinical Specialist Team, uMgungundlovu District, KwaZulu-Natal, South Africa \\ ${ }^{2}$ Section of Infectious Disease, Department of Medicine, Yale University School of Medicine, New Haven, Conn., USA \\ ${ }^{3}$ Department of Internal Medicine, Edendale Hospital, Pietermaritzburg, South Africa
}

Corresponding author: T P Kerry (tim.kerry@kznhealth.gov.za)

\begin{abstract}
Background. The correct antiretroviral (ARV) management of HIV-positive pregnant women can be complex, with many variables to consider. uMgungundlovu District Management in KwaZulu-Natal (KZN) Province, South Africa, suspected that the taking and retrieval of blood tests relating to ARV management in pregnancy could be improved. An antenatal ARV tracking form was therefore designed: a dedicated page on which to write all the ARV-related blood results and remind clinicians to take the correct bloods at the appropriate time. The form was photocopied and distributed to all antenatal clinics throughout uMgungundlovu District.

Objectives. To audit uptake of use of the tracking form by antenatal clinicians, and to audit quality of care - did using the tracking form prompt the correct bloods to be taken and retrieved at the right time, and with correct antiretroviral management and outcome?

Methods. Data were collected by retrospective chart review of maternity case records of women who delivered at Northdale District Hospital, uMgungundlovu District, KZN. We determined compliance with use of the tracking form in the maternity case records of all HIVpositive women delivering at the hospital, and assessed quality of care by scoring 100 records of women with a tracking form in comparison with 100 records of women without one.

Results. District-wide uptake of use of the tracking form in maternity case records was 95\%. Quality of care showed a statistically significant improvement in women with a tracking form present in their maternity case record: nearly $80 \%$ of those with a form were correctly managed at the booking visit, v. $<20 \%$ of those without one $(79 \%$ v. $19 \%$; $p<0.001)$. The differences in quality of care persisted at 3 and 6 months after booking. In terms of outcome, HIV viral load was suppressed at the time of delivery in $86 \%$ of women with a tracking form v. $65 \%$ of those without, and $5 \%$ of women with a tracking form had an unknown viral load v. $21 \%$ of those without.

Conclusions. Uptake of use of the tracking form increased markedly after implementation. Use of the form significantly improved the taking and retrieval of blood tests and the management of ARVs. The form has since been printed as part of the new maternity case records in KZN.

S Afr Med J 2020;110(12):1206-1212. https://doi.org/10.7196/SAMJ.2020.v110i12.14505
\end{abstract}

The drive for optimal healthcare of pregnant women is one of the bases of the health system in South Africa (SA). Part of this responsibility concerns care of pregnant women who are living with HIV. Prevention of mother-to-child transmission (PMTCT) of HIV concerns reducing transmission of HIV to the fetus and infant.

The vast majority of pregnant women in KwaZulu-Natal (KZN) Province receive their antenatal care in primary healthcare (PHC) clinics, both fixed and mobile. In the PHC clinics, nurse-clinicians do almost all the work of screening pregnant women for HIV at their first antenatal or booking visit. The nurse-clinicians then take the baseline bloods that relate to pregnancy and HIV. HIV-positive pregnant women start taking antiretrovirals (ARVs) the same day. This booking visit is a complex process, in the course of which the nurse-clinician has to consider several variables, obstetric, HIVrelated and more. Often nurse-clinicians with relatively little ARV training and support have to undertake the task of ARV management of pregnant women.

The excellent design of the SA maternity case record (MCR) assists in this complex booking process by providing a well-laid-out document for antenatal care, particularly on pages 2 and 3. ${ }^{[1]}$ Page 2 contains fields for the booking visit. Page 3 contains the symphysisfundal height graph that allows monitoring of fetal growth, together with blood pressure, urinalysis, haemoglobin, etc. The MCR is a patient-held record and therefore bridges the communication gap antenatally between PHC clinic and hospital. Women are referred to a hospital-based high-risk antenatal clinic as necessary. Most women in KZN and SA deliver their babies in hospital labour wards. Postnatally, the communication gap between the hospital and the PHC clinic is bridged by the discharge summary, which is printed on pages 31 and 33 of the MCR. The top copy of the summary is taken home by the new mother. This summary provides vital information for the clinicians at primary care level who follow up the mother in the puerperium, as well as the new baby.

However, the SA MCR was not able to keep pace with recent advances in the national ARV programme. The MCR had a dedicated space only for the CD4 count on page 2, with no dedicated space for HIV viral load and serum creatinine results. Paper copies of the laboratory results were often stapled to the back of the MCR. According to the protocol in the national ARV guidelines at the time, ${ }^{[2]}$ blood tests were to be done as follows:

- At booking, a CD4 count was taken on all HIV-positive pregnant women. If the CD4 count was $<100$ cells $/ \mu \mathrm{L}$, disseminated cryptococcosis should be considered; a low CD4 count may also indicate the need for cotrimoxazole prophylaxis. Serum creatinine should have been taken at booking to check renal function, as tenofovir (potentially nephrotoxic) is part of the fixed-dose combinations (FDCs) of ARVs that most pregnant women take. The CD4 and creatinine results should have been 
reviewed the following week. A viral load should have been taken at booking if a woman was already taking ARVs before the pregnancy.

- At 3 months after booking, an ARV-naive pregnant woman should have had a viral load and creatinine taken.

- At 6 months after booking, all pregnant women on ARVs should have had a viral load and creatinine taken. The maternal HIV viral load should have been suppressed $(<1000$ copies $/ \mathrm{mL})$ before or by the time of delivery. The 3- and 6-month blood results were reviewed at the next antenatal visit.

Having a known and suppressed viral load was therefore the ideal situation for the woman to be in at the time of delivery. Many pregnant women were delivering without the hospital knowing their viral load. The hospital may have taken a viral load at time of delivery, but because the result takes a week to be available from the laboratory, it was not ready before the new mother was discharged and sent home. PHC clinics struggled to trace laboratory tests that were taken at the hospital.

The uMgungundlovu District Clinical Specialist Team (DCST) and District Management were concerned about the taking and retrieval of blood tests relating to ARV management in pregnancy, suspecting that it could be improved. The clinicians in the DCST 'provide clinical mentorship and guidance to health facilities to improve their ability to provide effective maternal, neonatal and child health services'. ${ }^{[3]}$ Improvements required were that the correct blood test needed to be taken, and at the appropriate time in relation to ARV initiation. The presence of a laboratory printout of the blood results in the MCR did not guarantee that the correct management was being undertaken.

\section{The antenatal ARV tracking form}

A group of nurses and a doctor from the DCST and Northdale Hospital met in 2015 to find a solution to the above problem (other resources examined included the PMTCT checklist that was used in Western Cape Province). Northdale is a busy district hospital in Pietermaritzburg, and delivered 5739 women in 2017. The group designed an antenatal ARV tracking form (Fig. 1) for easy use by nurse- and medical clinicians in the management of HIV-positive pregnant women. The tracking form was used at all PHC clinics and community health centres (CHCs) and in the four general hospitals

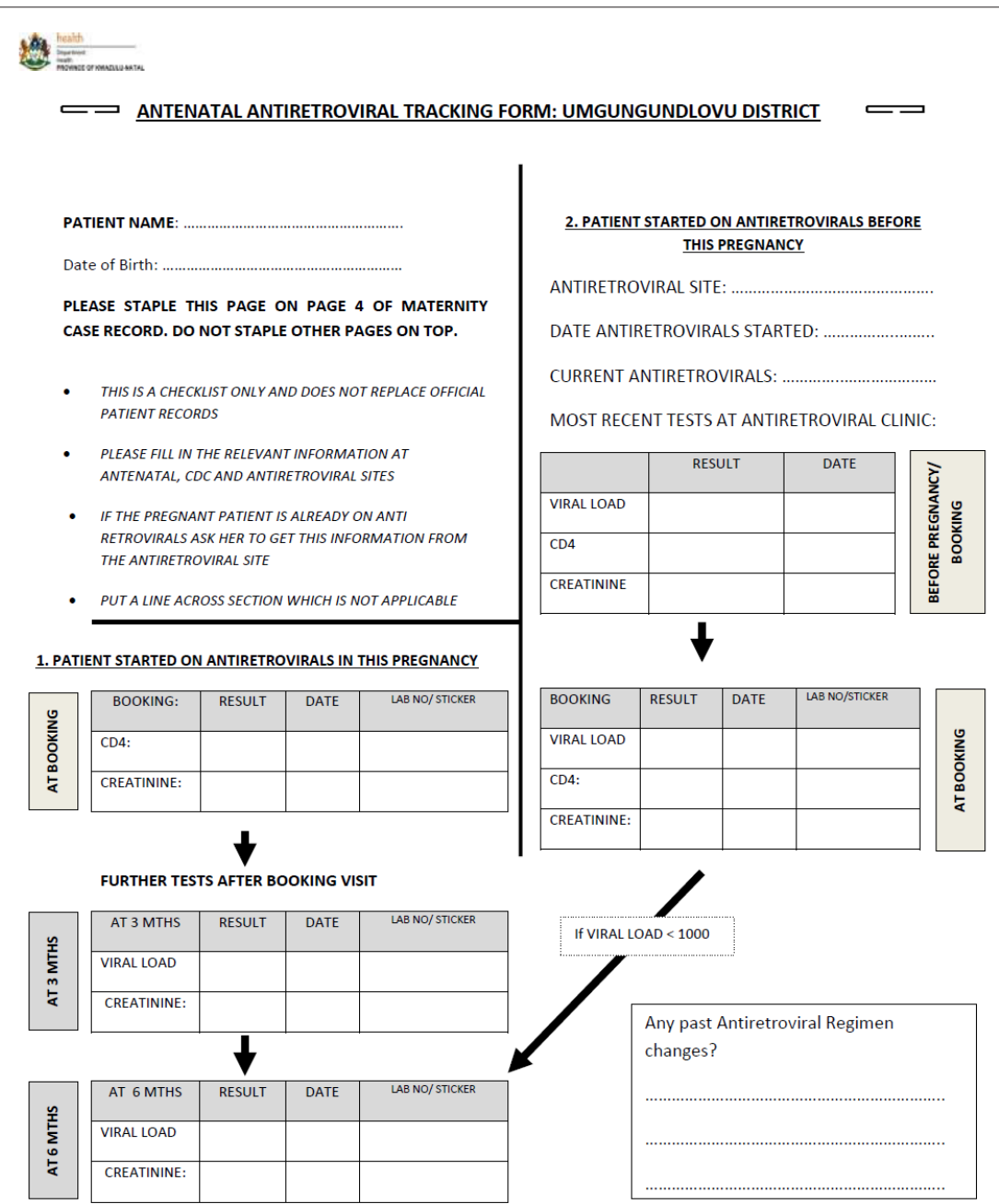

THE GOAL IS TO HAVE AN UNDETECTABLE AND RECORDED VIRAL LOAD BY THE TIME OF BIRTH

Fig. 1. The antenatal antiretroviral tracking form.

in uMgungundlovu District. The form was designed to be stapled onto page 4 of the MCR, which was the first blank writing page. The information was therefore prominently placed in the MCR and the form was easy to read and write on.

The aims of introducing the ARV tracking form were two-fold: $(i)$ to provide a dedicated and easy-to-use page on which to write all the required blood tests relating to ARVs in pregnancy; and (ii) for the form to serve as a reminder to do the correct bloods at the appropriate times: at booking, and at 3 and 6 months.

The ARV tracking form was introduced in August 2015 along with orientation sessions at perinatal mortality meetings and antenatal clinics throughout the district. An audit of this pilot phase of use of the tracking form was done in September 2016. During this month, 154 MCRs of HIV-positive pregnant women were checked in the postnatal ward at Northdale Hospital. Only $50 \%$ of MCRs had a tracking form. A more intensive campaign was therefore launched to ensure that all clinicians working in antenatal clinics used a tracking form in the MCR of every HIVpositive pregnant woman.

Anecdotally, the tracking form appeared to have been very effective in reminding clinicians to take and record the correct bloods at the correct time. However, a formal audit was required. The question was asked: 'Has the use of the ARV tracking form improved the monitoring of the relevant blood tests, leading to the correct ARV management of HIV-positive pregnant women?' In 2017, we obtained ethical clearance for the research from uMgungundlovu Health Ethics Review Board and with kind permission from Northdale Hospital. 


\section{Further background to the ARV tracking form}

The HIV prevalence among pregnant women in uMgungundlovu in 2017 was $>40 \%{ }^{[4]}$ The PHC nurse-clinicians were therefore working in a setting with a high prevalence of HIV. Quality of care and ARV management mainly related to the correct taking, retrieval and interpretation of the blood tests. The term 'management' includes dispensing the actual ARV pills by the clinician; however, this had become very simple, because a single daily FDC pill was used for most pregnant women. At the time of the research, clinicians were using the 2015 National Consolidated Guidelines. ${ }^{[2]}$ The guidelines were clear and simple to follow for the majority of pregnant women, who were treated with FDC ARVs, usually TEE (tenofovir, emtricitabine, efavirenz). There was also an application for smartphones and electronic tablets, so that the guidelines can easily be referenced in the consulting room. ${ }^{[5]}$ A recent review article supported the effectiveness of the 2015 ARV regimen for pregnant women. ${ }^{[6]}$ Recent updates of the ARV and PMTCT guidelines were released in 2019. ${ }^{[7,8]}$ The new guidelines included some changes to the timing of blood tests, especially an HIV viral load at the time of delivery. Dolutegravir, an integrase inhibitor, was added to first-line ARV regimens, including pregnant women from 8 weeks' gestation. uMgungundlovu District started using dolutegravir in December 2019, mainly in an FDC called TLD (tenofovir, lamivudine and dolutegravir).

The National Guidelines for Maternity Care, ${ }^{[9]}$ which are widely used in PHC clinics, CHCs and hospitals, clearly explain the necessary steps to be taken when starting a pregnant woman on ARVs. In SA, midwives initiate ARVs in the majority of the low-risk pregnant women, and doctors are involved in the management of women who have obstetric, renal or mental health problems or virological failure. Stinson et al. ${ }^{[10]}$ commented that the FDCs have made ARV therapy easier for both clinicians and (pregnant) women. ${ }^{[10]}$ The adaptation of adherence counselling led to shorter sessions that allow for same-day initiation, with increased post-initiation counselling being piloted in Khayelitsha, Cape Town. Despite the increased workload, midwives felt empowered and were passionate about their new role: 'Nowadays we manage their pregnancy and we also manage their HIV.' Stinson went on to comment: 'The new challenge is to retain women on ART [antiretroviral therapy] and achieve sustained virological suppression. The tracking form was designed to track the HIV viral load, at least during the antenatal course.

The MCR at the time of the research (2017) was a relatively new National Department of Health document, released in 2010. ${ }^{[1]}$ Sibiya et al. ${ }^{[1]}$ described the perceptions of nurses working in eThekwini District in KZN regarding the new MCR. There were several negative conclusions, including that 'They [the PHC nurses] stated that the card is not user-friendly and does not provide spaces for recording relevant elements for Basic Antenatal Care and PMTCT. Ngxongo et al. ${ }^{[12]}$ examined the application of the basic antenatal care principles in the MCR, also in eThekwini District. They wrote: 'It is recommended that relevant policies, service delivery guidelines, and protocols should be available in all PHC clinics that provide ANC services and all staff members and managers should be trained to use these documents to ensure safe and standardised practice. Records should be designed so that relevant aspects of the implementation of policies required are recorded. This will facilitate auditing of the implementation of policies.' The antenatal ARV tracking form was designed to achieve this recommendation.

\section{Objectives}

The first objective of the research was to audit uptake of use of the antenatal ARV tracking form by antenatal clinicians. The second objective was to audit the quality of care - did use of the tracking form prompt the correct bloods to be taken and retrieved at the correct time, and with correct ARV management and outcome?

\section{Methods}

The data for the research audit were retrieved from the MCRs of HIVpositive pregnant women who either delivered or were managed in the postpartum ward at Northdale Hospital and had been discharged home. The research design was a retrospective chart review.

The MCRs of HIV-positive patients were audited as follows:

- Uptake of use of the antenatal ARV tracking form by antenatal clinicians was measured. The MCRs of all HIV-positive patients who delivered at Northdale Hospital during December 2017 were checked for the presence and use of a tracking form.

- Quality of care was then determined by checking 100 patients' MCRs with a tracking form and 100 patients' MCRs without a form. The 100 MCRs with a tracking form were identified by working back in time from 31 December, using the date of delivery. The 100 MCRs without a form were identified from the whole of 2017 and January 2018. A longer time period of data collection had to be used because of the scarcity of MCRs of HIV-positive women without a tracking form in their MCR. (Based on the HIV prevalence of $40 \%$ and the fact that only $5 \%$ of MCRs did not have a tracking form, few MCRs were suitable.)

A 1-page tracking form audit tool (Fig. 2) was developed to capture the results from the MCR of each woman. The following data were audited:

- general information about the pregnancy, e.g. gestation at booking

- CD4, creatinine and possibly viral load at booking

- viral load and creatinine after 3 months if the woman was ARV naive

- viral load and creatinine after 6 months

- retrieval, recording and interpretation of the blood results at the next scheduled antenatal visit

- correctness of ARV management at booking, 3 months and 6 months

- HIV viral suppression at the time of delivery.

\section{Exclusions}

The following women were excluded from the study: $(i)$ women who started their ARVs $<1$ month before delivery; (ii) 'unbooked' women who had not attended antenatal care; (iii) women who attended antenatal care in other southern African countries, as these countries may have had a different PMTCT programme; and (iv) women who delivered babies at $<28$ weeks' gestation by dates and where there was no evidence of life at delivery.

\section{Statistical analysis}

The data in the 200 audit tools were captured onto Excel spreadsheets, version 2010 (Microsoft, USA). We then analysed baseline characteristics of the women by univariate comparisons. We used the $\chi^{2}$ test for categorical variables, the one-way test for continuous variables, and the Kruskal-Wallis test for non-normal continuous variables (weeks' gestation at delivery). ${ }^{[13,14]}$ Statistical analysis was done with the $\mathrm{R}$ statistical computing environment, version 3.6.0 (R Foundation for Statistical Computing, Austria) and the package 'tableone. ${ }^{[15,16]}$

\section{Results}

\section{Uptake of tracking form use}

Of the $152 \mathrm{HIV}$-positive patients who delivered at Northdale Hospital during December 2017, 144 had an antenatal ARV tracking form 


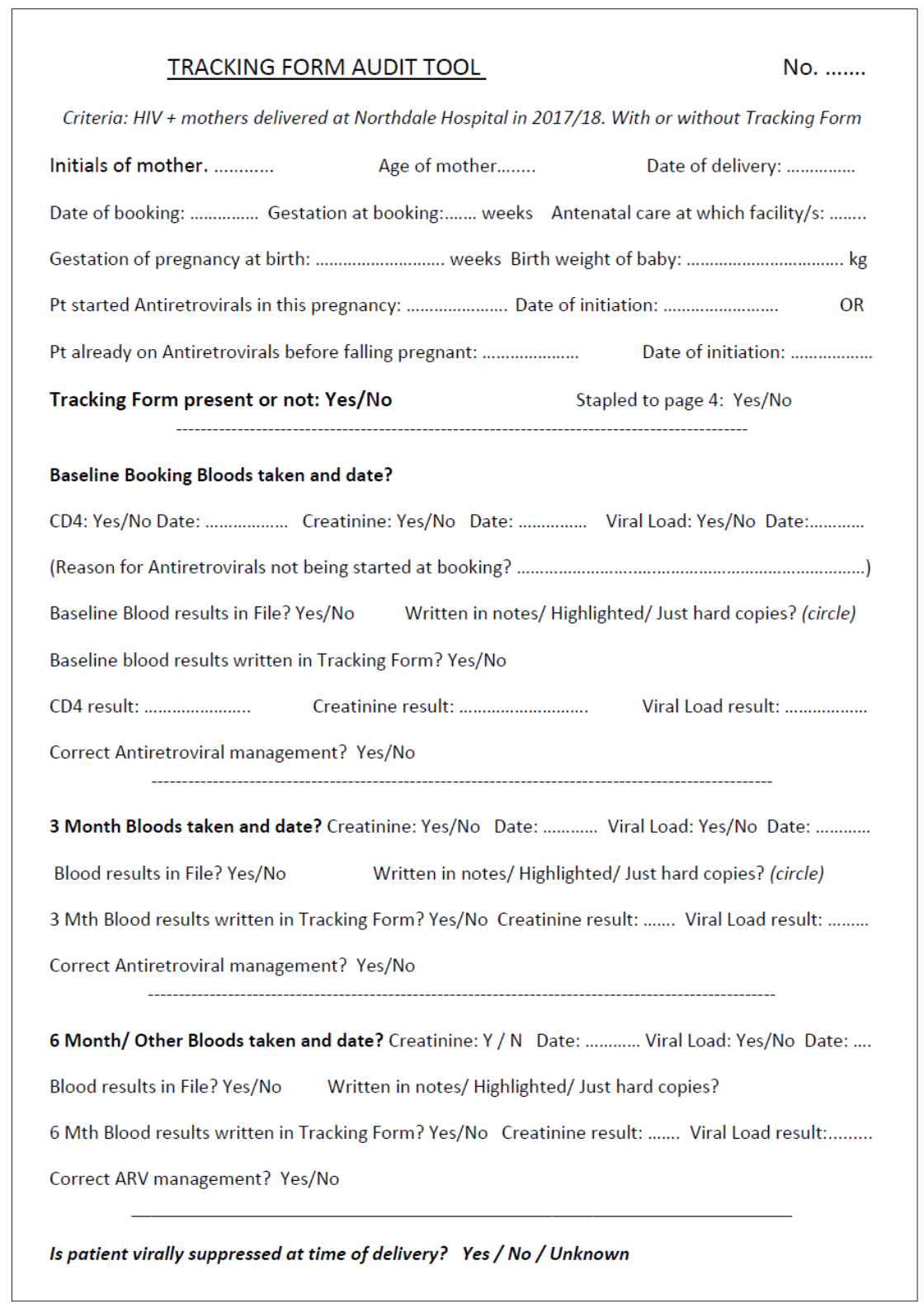

Fig. 2. Antiretroviral tracking form audit tool.

inserted and utilised in their MCR (95\% compliance).

\section{Baseline characteristics of the patients (Table 1)}

The baseline characteristics of the 100 women with a tracking form in their MCR and the 100 women without one showed no significant differences in maternal age, gestation at booking or delivery, infant birthweight, or the timing of ARV initiation before or during their pregnancies. Of the women without a tracking form in their MCR, $62 \%$ had booked in uMgungundlovu: conversely, $38 \%$ had booked in another district in KZN or SA and yet had delivered at Northdale Hospital. showed no significant differences in maternal age, gestation at booking or delivery, infant birthweight, or the timing of ARV initiation before or during the pregnancy.

\section{Quality of care (Table 3)}

Outcomes between patients with and without a tracking form present in the MCR were statistically different by univariate analysis. Nearly $80 \%$ of women with a tracking form in their MCR were correctly managed at the booking visit, v. $<20 \%$ of those without the form $(79 \%$ v. $19 \% ; p<0.001)$. The differences in quality of care persisted at 3 and 6 months after booking.

Women without a tracking form in their MCR were also more likely to have errors in ARV management ( $92 \%$ v. $38 \%$; $p<0.001$ ) and renal function monitoring ( $86 \%$ v. $32 \%$; $p<0.001)$.

Most notably, tracking form use was significantly associated with having a suppressed maternal HIV viral load at delivery ( $86 \%$ v. $65 \% ; p=0.001)$. The key results are summarised graphically in Fig. 3.

\section{Tracking form placement in the MCR (Table 4)}

When we looked at the effects of correct placement of the tracking form on page 4 of the MCR in the subgroup of women with a form, correct placement was associated with fewer errors in ARV management (29\% v. $54 \% ; p=0.025)$ and renal monitoring $(21 \%$ v. $51 \% ; p=0.005)$. However, there was no significant difference in maternal HIV viral load suppression at delivery $(88 \%$ v. $83 \%$; $p=0.812$ ).

\section{Qualitative analysis of the MCRs}

Some issues of quality were noted during the analysis of the 200 MCRs.

The recommended follow-up dates were not always adhered to by clinicians or patients. There were several instances of bloods being taken, but the results not being retrieved. The following systems issues were identified from the clinicians' notes: (i) paper copies of the laboratory results were not received back at the clinic; (ii) clinic telephones were out of order so clinicians could not phone the laboratory for blood results (a very timeconsuming process anyway); (iii) clinicians could not access the blood results from the database on the National Health Laboratory Service (NHLS) Labtrak system because the clinic did not have intranet access, a Labtrak password or Labtrak access via their smartphone; (iv) mobile clinics could not review patients 1 week after booking because the mobile clinic only visited that point once 
Table 1. Baseline characteristics of the patients included in the audit

\begin{tabular}{|c|c|c|c|}
\hline Indicator & Tracking form present & Tracking form missing & $p$-value \\
\hline$N$ (total number of records) & 100 & 100 & \\
\hline Maternal age (years), mean (SD) & $28.88(5.97)$ & $27.63(6.13)$ & 0.146 \\
\hline Gestation at booking (weeks), mean (SD) & $16.77(7.02)$ & $18.55(8.23)$ & 0.103 \\
\hline Booked before 20 weeks' gestation, $n(\%)$ & $65(65.0)$ & $56(57.1)^{*}$ & 0.323 \\
\hline Weeks' gestation at delivery, median (IQR) & $39.0(38.0-40.0)$ & $40.0(37.0-40.0)$ & 0.688 \\
\hline Birthweight (g), mean (SD) & $3030(42)$ & $2980(52)$ & 0.439 \\
\hline Low-birthweight babies (<2 $500 \mathrm{~g}), n$ (\%) & $9(9.0)$ & $13(13.0)$ & 0.498 \\
\hline ARVs initiated during this pregnancy, $n(\%)$ & $36(36.0)$ & $40(40.0)$ & 0.662 \\
\hline ARVs initiated before this pregnancy, $n(\%)$ & $64(64.0)$ & $60(60.0)$ & 0.662 \\
\hline Booked at an uMgungundlovu District health facility, $n$ (\%) & $100(100)$ & $62(62.0)$ & $<0.001$ \\
\hline
\end{tabular}

Table 2. Tracking form placement in patients with a tracking form in their MCR

$N$ (total number of records)

Maternal age (years), mean (SD)

Gestation at booking (weeks), mean (SD)

Booked before 20 weeks' gestation, $n$ (\%)

Weeks' gestation at delivery, median (IQR)

Birthweight (g), mean (SD)

Low-birthweight babies (<2 $500 \mathrm{~g}), n$ (\%)

ARVs initiated during this pregnancy, $n(\%)$

ARVs initiated before this pregnancy, $n$ (\%)

Booked at an uMgungundlovu District health facility, $n(\%)$

Tracking form on p. 4

65

$28.43(6.28)$

16.37 (7.18)

$43(66.2)$

$39.0(38.0-40.0)$

$3020(38)$

5 (7.7)

27 (41.5)

38 (58.5)

$65(100.0)$
Tracking form not on p. 4 -value

35

$29.71(5.33) \quad 0.307$

$17.51(6.73) \quad 0.439$

$22(62.9) \quad 0.912$

$39.0(38.0-40.0) \quad 0.503$

$3040(49) \quad 0.837$

4 (11.4) $\quad 0.798$

$9(25.7) \quad 0.176$

$26(74.3) \quad 0.176$

$35(100.0) \quad n / a$

$\mathrm{MCR}=$ maternity case record; $\mathrm{SD}=$ standard deviation; $\mathrm{IQR}=$ interquartile range; $\mathrm{ARVs}$ = antiretrovirals; $\mathrm{n} / \mathrm{a}=$ not applicable

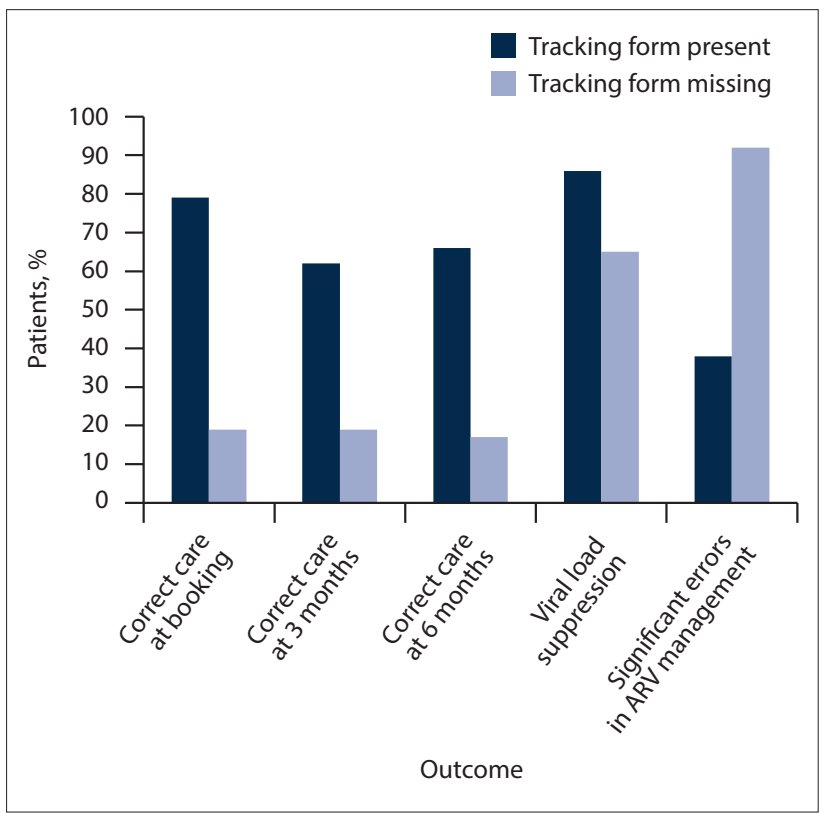

Fig. 3. Stratified outcomes: Quality of care in patients with and without a tracking form in their MCR. (MCR = maternity case record; $A R V=$ antiretroviral.)

a month; and $(v)$ there were incidences when blood results were not ready on time owing to rejection by the NHLS electronic gatekeeping (EGK) system. Once this happened, the nurse-clinicians working in the antenatal clinics struggled to catch up with the correct bloods (The NHLS has since introduced a special EGK code for the PMTCT situation that will obviate these delays.) The audit of the 200 MCRs showed that improvements could be made in the completion of the discharge summaries. During the working week, the regular PMTCT midwife included the pertinent results and comments for good ARVrelated follow-up at the PHC clinic. When the PMTCT midwife was not on duty, the quality of ARV-related information in the discharge summary was poorer.

\section{Discussion}

Uptake of tracking form use in antenatal clinics

Utilisation of the antenatal ARV tracking form in the MCRs had improved in uMgungundlovu District since implementation in August 2015. Uptake in use increased from 50\% to $95 \%$ in the 15 months between audits. This good level of compliance reflected well on the clinicians working in the antenatal clinics. Use of the tracking form had been actively promoted, but such good compliance may indicate that the form was actually assisting clinicians in their work in the antenatal clinics.

\section{Baseline characteristics of the pregnant patients}

There were no significant differences in baseline characteristics between women with or without a tracking form in their MCR. Similarly, there were no significant differences between those with the form placed correctly on page 4 or not. These findings suggest a lack of bias in those women who had a tracking form. In the group of pregnant women with a tracking form in their MCR, it followed that $100 \%$ 
Table 3. Stratified outcomes: Quality of care in patients with and without a tracking form in their MCR

\begin{tabular}{|c|c|c|c|}
\hline & Tracking form present & Tracking form missing & $p$-value \\
\hline$N$ (total number of records) & 100 & 100 & \\
\hline Correct care ${ }^{\star}$ at booking, $n(\%)$ & $79(79.0)$ & $19(19.4)^{\dagger}$ & $<0.001$ \\
\hline Correct care ${ }^{\star}$ at 3 months, $n(\%)$ & $32(61.5)^{\ddagger}$ & $11(19.0)^{\ddagger}$ & $<0.001$ \\
\hline Correct care $^{\star}$ at 6 months, $n(\%)$ & $23(65.7)^{\ddagger}$ & $6(17.1)^{\ddagger}$ & $<0.001$ \\
\hline Significant errors ${ }^{\S}$ in ARV management, $n(\%)$ & $38(38.0)$ & $92(92.0)$ & $<0.001$ \\
\hline Significant errors in renal monitoring, $n(\%)$ & $32(32.0)$ & $86(86.0)$ & $<0.001$ \\
\hline Maternal HIV viral load suppressed ( $<1000$ copies/mL) at delivery, $n(\%)$ & & & 0.001 \\
\hline No & $9(9.0)$ & $14(14.0)$ & \\
\hline Yes & $86(86.0)$ & $65(65.0)$ & \\
\hline Unknown & $5(5.0)$ & $21(21.0)$ & \\
\hline \multicolumn{4}{|c|}{$\begin{array}{l}\text { MCR = maternity case record; ARV }=\text { antiretroviral. } \\
{ }^{*} \text { Correct bloods taken and retrieved at the correct time, and correct ARV management and outcome. } \\
\text { 'MCRs missing a tracking form: } 98 \text { of } 100 \text { were analysed at booking. The two gaps in ARV-related bloods were as follows: one woman seroconverted during pregnancy, and the private doctor did } \\
\text { not write the blood results in the MCR; and one MCR was lost by a patient who had social problems, so another MCR was issued. } \\
\text { "The denominators for women at } 3 \text { and } 6 \text { months decreased from booking for the following reasons: some women delivered before the 3- or } 6 \text {-month bloods became due; and women already on } \\
\text { ARVs did not require bloods to be taken at } 3 \text { months if their viral load was suppressed at booking. } \\
\text { 'Significant errors included the following examples: blood tests not taken at the correct time; blood results not retrieved; blood results such as high creatinine not acted upon; high viral load not } \\
\text { managed correctly or timeously; patient given an incorrect follow-up date, etc. }\end{array}$} \\
\hline
\end{tabular}

Table 4. Stratified outcomes based on tracking form placement in those patients with a form in their MCR

\begin{tabular}{llll}
\hline & Tracking form on $\mathbf{p .} \mathbf{4}$ & Tracking form not on p. $\mathbf{4}$ & $\mathbf{p}$-value \\
\hline$N$ (total number of records) & 65 & 35 & \\
Correct care at booking, $n(\%)$ & $54(83.1)$ & $25(71.4)$ & 0.268 \\
Correct care at 3 months, $n(\%)$ & $26(70.3)$ & $6(40.0)$ & 0.086 \\
Correct care at 6 months, $n(\%)$ & $17(77.3)$ & $6(46.2)$ & 0.132 \\
Significant errors in ARV management, $n(\%)$ & $19(29.2)$ & $19(54.3)$ & 0.025 \\
Significant errors in renal monitoring, $n(\%)$ & $14(21.5)$ & $18(51.4)$ & 0.005 \\
Maternal HIV viral load suppressed $(<1000$ copies $/ \mathrm{mL})$ at delivery, $n(\%)$ & & 0.812 \\
$\quad$ No & $5(7.7)$ & $4(11.4)$ & \\
$\quad$ Yes & $57(87.7)$ & $29(82.9)$ & \\
$\quad$ Unknown & $3(4.6)$ & $2(5.7)$ &
\end{tabular}

booked at an antenatal clinic in uMgungundlovu District (at that time, uMgungundlovu was the only district using the tracking form). Of the group of women without a tracking form, $38 \%$ had booked in another district or province in SA. It is known that a significant percentage of patients in KZN are geographically mobile. ${ }^{[17]}$ The use of the patientheld MCR does assist the continuity of information.

\section{Quality of care}

Our analysis showed a clear improvement in correctness of care at booking and at 3 and 6 months of pregnant women with a tracking form present in their MCR, in comparison with women without a form. There was a significant improvement in maternal HIV viral load suppression at the time of delivery in women with a tracking form. Several factors (e.g. individual, social, pharmacological and financial) contribute to HIV viral suppression in pregnant women. However, clinicians should optimise quality of healthcare, which is but one of these factors. The use of tracking forms did assist the clinicians in improving quality of healthcare. It was a concern that 23 of the 200 women's viral loads (11.5\%) were unsuppressed, in spite of receiving antenatal care from clinicians at both PHC clinics and hospitals. A key advantage of the use of a tracking form was the reduction of unknown viral load results $(n=5 \mathrm{v} . n=21)$. A known viral load was important at the time of delivery, because the infant's PMTCT treatment differed if the viral load was $>1000$ copies $/ \mathrm{mL}$. A viral load could be taken at the hospital, but it may have been difficult to trace the result at primary care level, as not all PHC clinics had access to the NHLS Labtrak system.

\section{Placement of the tracking form in the MCR}

Ideally the tracking form should have been stapled onto page 4 of the MCR. Of the 100 tracking forms, 35 were stapled in other places in the MCR, especially to the inside front cover. This made it very difficult to find, update, refer to or even remember to use the tracking form.

\section{Significant errors in care}

The number of significant errors in general ARV care was lower in women whose MCR had a tracking form than in women without a form. Many of the significant errors were related to the taking, checking and interpretation of blood results. The number of significant errors with renal function monitoring was also lower in women with a tracking form present in their MCR. In pregnancy, the serum creatinine level is used to measure renal function in the clinic setting, the cut-off being $85 \mathrm{mmol} / \mathrm{L}$.

\section{Antenatal tracking form and discharge summaries}

Antenatally, the tracking form has helped bridge the HIV information gap that exists between primary care and hospital level. Postnatally, the information gap from hospital level back to PHC clinic should be bridged by the discharge summary. Discharge summaries could have 
been more clearly and accurately written. Better information about HIV and postnatal ARV care could be integrated into a new format of the discharge summary.

\section{Study limitations}

The 100 MCRs with a tracking form were sampled in December 2017. The 100 MCRs without a tracking form required a longer period of sampling because of the scarcity of usable MCRs. There was overlap of the time periods, but the periods did differ.

\section{Conclusions}

The use of the antenatal ARV tracking form met the two aims. Firstly, the form provided a dedicated, easy-to-use page on which to write all ARV-related blood tests in pregnancy - the form was well utilised by the antenatal clinicians. Secondly, the form provided a reminder to clinicians to do the correct bloods at the appropriate time. The use of the tracking form in the MCRs improved the taking and retrieval of the relevant blood tests, leading to significantly better ARV management of HIV-positive pregnant women.

\section{Recommendations}

- Some type of antenatal ARV tracking form should be incorporated in the future design of the MCR. The KZN Department of Health has adopted the use of the tracking form, and from 2019 all newly printed MCRs have a tracking form printed on page 4.

- Minor modifications may have to be made to the tracking form so that it aligns with the new 2019 PMTCT guidelines. ${ }^{[1]}$

- To strengthen the postnatal link between hospital level and follow-up of ARV management and blood results at PHC level, better information about HIV and postnatal ARV care could be integrated into the format of the discharge summary in the future design of the MCR.

- All fixed and mobile PHC clinics should have access to laboratory results on NHLS Labtrak, either by computer or smartphone. This will facilitate the tracing of the important laboratory tests relating to HIV in pregnancy: HIV viral load, CD4, serum creatinine, Xpert for tuberculosis, and HIV polymerase chain reaction in babies.

\section{Declaration. None.}

Acknowledgements. Thanks to Northdale Hospital and the KwaZulu-Natal Department of Health for their support.
Author contributions. TPK was part of the team that designed the tracking form. He conceptualised the research, conducted data collection and initial data analysis, and wrote the bulk of the article. PGTC conducted the statistical analysis and statistical comments and made comments as co-author. Funding. None. The audit was part of the routine Department of Health business in uMgungundlovu.

Conflicts of interest. TPK was part of the group that designed the antenatal ARV tracking form. No other conflicts of interest.

1. National Department of Health, South Africa. Maternity Case Record. Pretoria: DoH, 2010.

2. National Department of Health, South Africa. National Consolidated Guidelines for PMTCT and the Management of HIV in Children, Adolescents and Adults. Pretoria: DoH, April 2015. http://www.health.gov.za/index.php/2014-03-17-09-09-38/policies-and-guidelines/category/2302015p?download=937: national-art-guidelines-2015final (accessed 2015)

3. National Department of Health, South Africa. Handbook for District Clinical Specialist Teams. Pretoria: NDoH, 2014. https://www.hst.org.za/publications/HST\%20Publications/Clinical\%20Gov\%20 Handbook_LR_24Oct2014.pdf (accessed 2014).

4. National Department of Health, South Africa. The 2017 National Antenatal Sentinel HIV Survey. Pretoria: NDoH, 2018. https://www.nicd.ac.za/wp-content/uploads/2019/07/Antenatal_surveyreport_24July19.pdf (accessed 2019).

5. National Department of Health, South Africa and Open Medicine Project. HIV Clinical Guide (app for smartphone or e-tablet), 2015.

6. Meintijes G, Moorehouse MA, Carmona S, et al. Adult Antiretroviral Therapy Guidelines 2017. South Afr Meintiies G, Moorehouse MA, Carmona S, et al. Adult Antiretroviral Ther
J HIV Med 2017;18(1):a776. https://doi.org/10.4102/sajhivmed.v18i1.776

7. National Department of Health, South Africa. 2019 ART Clinical Guidelines for the Management of HIV in Adults, Pregnancy, Adolescents, Children, Infants and Neonates. Pretoria: NDoH, October 2019. https://www.knowledgehub.org.za/elibrary/2019-art-clinical-guidelines-management-hiv-adultspregnancy-adolescents-children-infants (accessed 2019).

8. National Department of Health, South Africa. Guideline for the Prevention of Mother to Child Transmission of Communicable Diseases. Pretoria: NDoH, October 2019. https://www.nicd.ac.za/wp content/uploads/2019/11/Guidelines-for-the-Prevention-of-Transmission-of-Communicable-Diseasesfrom-mother-to-child_28-October.pdf (accessed 2019).

9. National Department of Health, South Africa. Guidelines for Maternity Care in South Africa: A Manual for Clinics, Community Health Centres and District Hospitals. 4th ed. Pretoria: NDoH, 2016. https:// www.knowledgehub.org.za/elibrary/guidelines-maternity-care-south-africa-2016 (accessed 2016).

10. Stinson K, Giddy J, Cox V et al. Reflections on a decade of delivering PMTCT in Khayelitsha, South Africa. South Afr J HIV Med 2014;15(1):30-32. https://doi.org/10.7196/sajhivmed.1025

11. Sibiya MN, Cele RJ, Ngxongo P. Assessment of the use of the new maternity case record in improving the quality of antenatal care in eThekwini District, KZN. Int J Afr Nurs Sci 2015;2:53-58. https://doi.
the the quality of antenatal care in
org/10.1016/ji.jans.2015.04.002

12. Ngxongo TSP, Sibiya MN, Gwele NS. Evidence of application of the basic antenatal care principles of good care and guidelines in pregnant women's antenatal care records. Afr J Prim Health Care Fam Med 2016;8(2). https://doi.org/10.4102/phcfm.v8i2.1016

13. Welch BL. On the comparison of several mean values: An alternative approach. Biometrika 1951;38(3/4):330-336. https://doi.org/10.2307/2332579

14. Kruskal WH, Wallis WA. Use of ranks in one-criterion variance analysis. J Am Stat Soc 1952;47(260):583621. https://doi.org/10.2307/2280779

15. R Core Team. R: A language and environment for statistical computing. Vienna: R Foundation for Statistical Computing, 2019. https://www.scirp.org/(S(351jmbntvnsjtlaadkposzje))/reference/ ReferencesPapers.aspx?ReferenceID=2600003 (accessed 2019).

16. Yoshida K. tableone: Create 'Table I' to describe baseline characteristics 2020. R package version 0.11.1. https://CRAN.R-project.org/package=tableone (accessed 2020).

17. Du Plessis JG. Are patients prepared to pay for a patient retained medical record? MFamMed dissertation.

Pretoria: University of Pretoria, 1997.

Accepted 11 June 2020 